\title{
A close look at acid reflux drugs points to possible risks
}

For tens of millions of people, antacid tablets aren't enough to quell the burning chest pains from acid reflux. As a result, many turn to powerful proton pump inhibitors (PPIs) such as Nexium and Prevacid. Global sales of PPIs tallied \$25 billion last year, according to the market consultancy firm IMS Health. Now, a growing body of evidence suggests that such drugs might have previously unknown possible side-effects.

Recent preliminary research has linked high doses of PPIs to increased risk of bone fracture and pneumonia. A preliminary study published in July suggests that the drugs might perhaps induce the very symptoms they're supposed to treat (Gastroenterology, doi:10.1053/j. gastro.2009.03.058; 2009).

"Proton pump inhibitors are in many ways considered a panacea," says Kenneth McColl, a University of Glasgow gastroenterologist. "But I think we're becoming more aware that they aren't the ideal drug."

PPIs shut down pumps that parietal cells in the stomach rely on to make acid. Whereas antacids such as Tums and Rolaids neutralize acid, providing some temporary relief, one PPI tablet a day treats certain upper gastrointestinal ailments such as dyspepsia and gastroesophageal reflux disease.

"Because they're effective, safe and easy to use, an increasing number of patients are being prescribed PPIs," says Peter Bytzer, head of gastroenterology at Glostrup University Hospital, Copenhagen, and coauthor of the chronic users is increasing, which is difficult Gastroenterology study. "The number of

to understand because some of the underlying diseases PPIs are indicated for, especially peptic ulcer disease, are on the decline."

There has been some evidence suggesting that PPIs can sometimes cause rebound acid hypersecretion, a condition in which the stomach temporarily produces more acid after treatment than before (Gut 39, 649-653; 1996; Gastroenterology 116, 239-247; 1999; Basic Clin. Pharmacol. Toxicol. 94, 202-208; 2004). However, the clinical importance of this potential condition was uncertain. Bytzer's team conducted a small, preliminary study involving 120 adult participants with no history of acid reflux symptoms. In a double-blind trial they gave half of the subjects a placebo for 12 weeks and the other half a PPI, esomeprazole (Nexium), for eight weeks followed by placebo for four weeks. Forty-four percent of subjects in the drug group reported heartburn, dyspepsia or acid regurgitation while on placebo the final three weeks of the trial, compared with $15 \%$ of those on placebo the entire time.

"This study certainly changes my perception," says University of Pennsylvania epidemiologist Yu-Xiao Yang. "We always thought rebound hypersecretion was short lived-a few days. This study shows that people might have symptoms due to rebound that persist." Yang suggests that people with these rebound symptoms might go back on the drug.

Blair Hains, a spokesperson for AstraZeneca Pharmaceuticals, which provided the study drug, stressed that the trial looked only at healthy volunteers. "Whether the conclusion can be carried over to patients, we don't

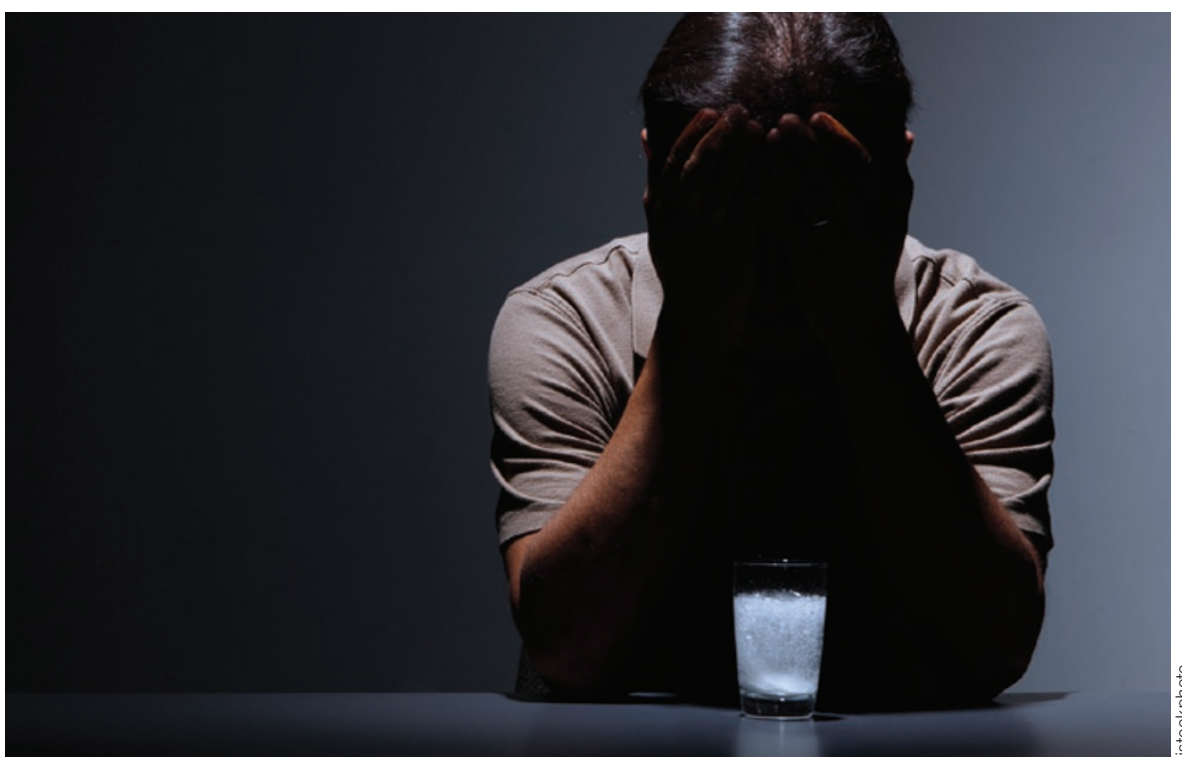

Burning question: Doctors seek answers about how to best treat acid reflux know." Bytzer plans to repeat the study in people with acid-related symptoms. Takeda Pharmaceuticals, the maker of Prevacid, declined to comment when contacted by Nature Medicine.

In the elderly, long-term use of PPIs has been tied to low bone mass and bone fracture. In 2006, Yang's group examined records of 13,556 people with hip fractures and 135,386 without, all aged 50 or older (J. Am. Med. Assoc. 296, 2947-2953; 2006). The study found that those taking PPIs for more than a year had a $44 \%$ higher risk of breaking a hip. Yang's group also found that high-dose PPI therapy was associated with a greater risk of hip fracture than therapy with regular doses.

Several groups have since reported similar findings, but questions remain. At the Digestive Disease Week conference in June, researchers from the University of Manitoba reported finding no increased risk of osteoporosis from chronic use of PPIs. Last year, however, they found that taking PPIs for seven years or more is associated with substantially increased risk of osteoporosis-related fracture (CMAJ 179, 319-326; 2008). "There's conflicting science," says Hain. "To be conclusive, we need to see prospective studies done."

Basic research has hinted that decreasing stomach acid lowers calcium absorption. An animal study published in June supports the connection (Nat. Med. 15, 674-682; 2009). Mice engineered to have neutral stomach $\mathrm{pH}$ developed low bone mass and, consequently, brittle bones. "Of course, we can't directly relate our findings to the human situation-we need clinical studies," says Michael Amling of the University Medical Center HamburgEppendorf, who led the study.

Community-acquired pneumonia has also been linked to PPI therapy. Yang found that long-term users had lower risk of pneumonia than those taking it for 30 days or less. "It's puzzling; you'd expect to find the opposite," he says. Yang believes the increased risk represents a bias-perhaps early pneumonia symptoms are mistakenly attributed to an acid-related condition, thereby prompting PPI therapy. "But," he says, "I probably do not represent most people in this regard."

Ultimately, PPIs should be prescribed at the lowest dose possible and histamine-2 antagonists and dietary changes should be considered, some experts say. "We need to be careful when we prescribe these very, very potent drugs and make sure that patients have a disease that needs PPI treatment," says Bytzer. Alisa Opar, New York 